\title{
Métodos recomendados para el estudio de susceptibilidad en Staphylococcus aureus, Staphylococcus coagulasa negativa y Staphylococcus saprophyticus: Nuevos puntos de corte e interpretación de resultados
}

\author{
ELIZABETH PALAVECINO R.*
}

\author{
RECOMMENDATIONS ON THE SUSCEPTIBILITY STUDIES OF \\ Staphylococcus aureus, COAGULASE NEGATIVE Staphylococcus AND \\ Staphylococcus saprophyticus:NEW BREAKPOINTS AND \\ INTERPRETATION OF RESULTS
}

Key words: Staphylococcus, Susceptibility testing, Breakpoint.

\section{INTRODUCCIÓN}

Los estafilococos son uno de los microorganismos más frecuentemente aislados en el laboratorio de microbiología. Aunque forman parte de la flora normal y en ciertas ocasiones pueden ser sólo contaminantes en una muestra, no hay duda que ellos causan infecciones severas, especialmente en el paciente hospitalizado.

Los estafilococos presentan mecanismos de resistencia a los antimicrobianos que son difíciles de detectar usando los métodos convencionales. Además, el NCCLS recientemente cambió los puntos de corte para oxacilina y por lo tanto, en este momento la interpretación de resistencia a oxacilina es diferente dependiendo si el microorganismo causante de la infección es Staphylococcus aureus o es Staphylococcus coagulasa negativa (SCN). Con estos nuevos puntos de corte es indispen- sable que la identificación de estas especies sea hecha en forma precisa y confiable.

Generalmente los laboratorios de microbiología utilizan la prueba de la coagulasa para diferenciar $S$. aureus de las otras especies de estafilococos que conforman los llamados SCN. Debe sin embargo, tenerse presente que cuando se usa solamente la prueba de la coagulasa rápida, la que detecta el clumping factor, ciertas especies dentro del grupo de SCN pueden dar una reacción positiva, es el caso de Staphylococcus lugdunensis y Staphylococcus schleiferi siendo erróneamente identificadas como $S$. aureus. Por fortuna estas especies son aisladas muy esporádicamente en muestras clínicas, aunque se sabe que pueden causar infecciones oportunistas. En mi experiencia, estas especies de SCN se comportan clínicamente más parecido a $S$. aureus y tal vez deberían ser interpretados con los puntos

\footnotetext{
* Department of Pathology, University Hospitals of Cleveland. Case Western Reserve University Cleveland OH, USA.
} 
de corte de $S$. aureus. Esto es todavía un punto de controversia y en la actualidad, si seguimos las guías del NCCLS, deben ser considerados como SCN y la oxacilina debe ser interpretada comotal.

La coagulasa en tubo es una prueba bastante específica para diferenciar entre $S$. aureus y SCN. Los métodos automatizados son en general bastante confiable en la identificación de estos microorganismos al nivel de especie. En la actualidad existen cerca de 20 especies de SCN pero habitualmente son consideradas un solo grupo. En la gran mayoría de los casos la identificación de especie no es necesaria, excepto para monitorear tendencias de los patrones de resistencia por motivos epidemiológicos o para diferenciar bacteremias verdaderas de contaminación en hemocultivos sucesivos. ${ }^{1}$ Aún hoy, Staphylococcus epidermidis es la especie más frecuentemente aislada de muestras clínicas y por lo tanto, la mayoría de las recomendaciones del NCCLS para los SCN están enfocadas a la evaluación de susceptibilidad de esta especie.

En este artículo describiremos las recomendaciones actuales del NCCLS para el estudio de susceptibilidad en estafilococos a oxacilina y a vancomicina así como también las nuevas interpretaciones y puntos de corte. ${ }^{2}$

\section{Estudio de susceptibilidad a oxacilina}

Los estafilococos son resistentes a oxacilina por tres mecanismos: producción exagerada de $\beta$-lactamasa, modificación de las PBPs, y por la presencia de una nueva proteína en la pared celular denominada PBP2a. Este último mecanismo es el más importante en las cepas que rutinariamente se aislan en el laboratorio. La presencia de PBP2a está codificada por el gen mecA. ${ }^{3}$ Las cepas que tienen este mecanismo presentan lo que se denomina una heterogeneidad dentro de la colonia. Esto significa que un porcentaje muy bajo de los clones dentro de la colonia son resistentes mientras que el resto de la población es susceptible. Además, los clones resistentes crecen más lento que los susceptibles, y por ello se requieren medios especiales y temperaturas no mayores de $35^{\circ} \mathrm{C}$ para estimular su desarrollo in vitro y poder detectarlos.
Es muy importante detectar en forma eficiente a los microorganismos resistentes dentro de esa población causante de la infección porque si el paciente es tratado con oxacilina, las cepas resistentes van a persistir en el sitio de la infección. Debido a la complejidad de esta resistencia el NCCLS ha desarrollado métodos que son específicamente diseñados para detectarla (Tabla 1).

El método considerado gold standard para identificar las cepas de Stafilococcus resistentes a oxacilina es la detección del gen mecA. El gen $m e c \mathrm{~A}$ codifica la formación de una PBP denominada PBP2a la que tiene una baja afinidad por meticilina y oxacilina y por lo tanto estas cepas son resistentes al tratamiento con estos agentes antimicrobianos ${ }^{3} \mathrm{El}$ gen mecA puede ser detectado por RPC usando los partidores que se han publicado previamente ${ }^{4} \mathrm{O}$ se puede usar una sonda de ADN que detecta la PBP2a. Sin embargo, estos métodos todavía no son usados de rutina en el laboratorio ya que por lo menos en E.U.A. no han sido aprobados por el FDA para el uso comercial.

La detección de resistencia se complica aún más ya que el gen mecA existe en todas las cepas de $S$. aureus con una CIM a oxacilina $>2 \mu \mathrm{g} / \mathrm{ml}$ y está ausente en aquellas cepas con una CIM $<2 \mu \mathrm{g} / \mathrm{ml}$. El punto de corte que el NCCLS había designado para oxacilina en Stafilococcus era de $2 \mu \mathrm{g} / \mathrm{ml}$. Sin embargo, estudios hechos en SCN demostraron que cepas que tenían una CIM de $0,5 \mu \mathrm{g} / \mathrm{ml}$ tenían el gen mecA y por lo tanto, deberían ser consideradas resistentes. ${ }^{4}$ Además, informes de fallas de tratamiento con estos agentes en infecciones producidas por SCN con CIM de 0,5 a 1 $\mu \mathrm{g} / \mathrm{ml}$ sugerían que estas cepas se comportaban clínicamente como resistentes a oxacilina. Por este motivo el año pasado el NCCLS decidió establecer diferentes puntos de corte para oxacilina en $S$. aureus y en SCN (Tabla 2).

Cuando se informa la susceptibilidad de estafilococo es necesario tener presente si se trata de $S$. aureus o de $\mathrm{SCN}$ e interpretar el resultado de la oxacilina de acuerdo a la especie. También es necesario recordar que todas las cepas de estafilococo que son resistentes a oxacilina (SAMR o SCNMR) deben ser informadas como resistentes a todos los $\beta$-lactámi- 
Nuevos puntos de corte e interpretación de resultados en Staphylococcus spp - E. Palavecino R.

Tabla 1. Métodos recomendados por el NCCLS para la detección de resistencia a oxacilina en el género Staphylococcus

\begin{tabular}{|c|c|c|c|}
\hline Microorganismo & Método & Medio & Control de calidad \\
\hline S. aureus & Placa de screen* & $\begin{array}{l}\text { Agar de Mueller-Hinton }+ \\
4 \% \mathrm{NaCl}+6 \mu \mathrm{g} / \mathrm{ml} \text { oxacilina }\end{array}$ & $\begin{array}{l}\text { S. aureus ATCC } 29213 \text {-Susceptible } \\
\text { S. aureus ATCC 43300-Resistente }\end{array}$ \\
\hline \multirow[t]{2}{*}{$\mathrm{SCN}++$} & Difusión por disco & Agar de Mueller-Hinton & S. aureus ATCC 29213 \\
\hline & Microdilución en caldo & $\begin{array}{l}\text { CAMHB *** } \\
2 \% \mathrm{Na} \mathrm{Cl}\end{array}$ & S. aureus ATCC 29213 \\
\hline $\begin{array}{l}* \text { El inóculo debe } \mathrm{se} \\
10 \mathrm{ml} \text { o una tórula } \\
\text { Para cada método } 1 \\
* * \text { Staphylococcus }\end{array}$ & $\begin{array}{l}\text { r preparado de suspensión } \\
\text { y se debe sembrar un área } \\
\text { incubación debe ser a } 35 \\
\text { coagulasa negativa. } * * * \text { C }\end{array}$ & $\begin{array}{l}\text { directa ajustado a } 0,5 \text { McFarland. } \\
\text { de } 10-15 \mathrm{~mm} \text { de diámetro o un } \\
\text { C durante } 24 \mathrm{~h} \text { antes de interp } \\
\text { AHB: Caldo de Mueller Hinto }\end{array}$ & $\begin{array}{l}\text { a la inoculación, se puede usar un asa de } \\
\text { drante de la placa. } \\
\text { r los resultados. } \\
\text { on ajuste de cationes. }\end{array}$ \\
\hline
\end{tabular}

cos y a las combinaciones de $\beta$-lactámicos con inhibidores de $\beta$-lactamasas.

\section{Métodos recomendados para detectar resistencia a oxacilina en $S$. aureus}

El método más práctico y confiable para detectar la resistencia a oxacilina en $S$. aureus es la placa de screening que contiene cloruro de sodio y oxacilina. En la Tabla 1 se detallan los requerimientos técnicos. Esta placa se inocula con una suspensión bacteriana con una densidad de 0,5 McFarland preparada disolviendo un par de colonias directamente en caldo de Mueller-Hinton. Se introduce una tórula de algodón en el tubo que contiene la suspensión de microorganismos, se exprime el exceso de líquido de la tórula en el borde del tubo y se siembra en una porción de la superficie de agar (la placa se puede dividir en ocho porciones). Las placas se deben incubar durante $24 \mathrm{hrs}$ a $35^{\circ} \mathrm{C}$ antes de informar una cepa como susceptible. Si una cepa demuestra resistencia a las 18 hrs (una o más colonias creciendo en el agar) se puede informar como tal, pero si no se observa crecimiento se debe incubar hasta completar las $24 \mathrm{hrs}$ antes de informar. Este método no necesita confirmación.

Los otros métodos recomendado por la NCCLS para detectar la resistencia a oxacilina en $S$. aureus son el método de dilución en agar de Mueller-Hinton con $2 \%$ de $\mathrm{NaCl}$ y el método de difusión por disco usando sólo una placa de agar de Mueller-Hinton ( $\sin \mathrm{NaCl})$. El método de difusión por disco detecta la mayoría de los SAMR pero en algunos casos el resultado necesita ser confirmado. Otro método confiable es el E-test ${ }^{\circledR}$ si se usa una placa de MuellerHinton con $2 \% \mathrm{NaCl}$.

Tabla 2. Nuevos puntos de corte para la oxacilina en Staphylococcus

\begin{tabular}{|c|c|c|c|c|c|c|}
\hline \multirow[t]{2}{*}{ Microorganismo } & \multicolumn{2}{|r|}{ CIM $(\mu \mathrm{g} / \mathrm{ml})$} & \multicolumn{3}{|c|}{ Área de inhibición } & \multirow{2}{*}{$\underset{\mathbf{R}}{(\mathbf{m m})}$} \\
\hline & $\mathbf{S}$ & I & $\mathbf{R}$ & $\mathbf{S}$ & I & \\
\hline S. aureus* & $<2$ & - & $>4$ & $>13$ & $11-12$ & $<10$ \\
\hline $\mathrm{SCN}$ & $<0,25$ & - & $>0,5$ & $>18$ & - & $<17$ \\
\hline \multicolumn{7}{|c|}{$\begin{array}{l}\text { * En } S \text {. aureus los resultados de difusión por disco o de la CIM se pueden confirmar usando la } \\
\text { placa de screen. En Staphylococcus coagulasa negativa la placa de screen no debe ser usada ya que } \\
\text { este método no detecta cepas de SCN resistentes a oxacilina. }\end{array}$} \\
\hline
\end{tabular}




\section{Métodos recomendados para detectar resistencia a oxacilina en Staphylococcus coagulasa negativa}

Aunque muchas veces es difícil determinar la significancia real de la presencia de una cepa de SCN en un cultivo de muestras clínicas, este grupo se ha convertido en un patógeno importante especialmente en infecciones intrahospitalarias. El aumento en la cantidad de procedimientos invasores y en el uso de catéteres han contribuido, sin duda, al aumento de infecciones causadas por SCN. En general $S$. epidermidis, Staphylococcus haemolyticus y Staphylococcus hominis son más resistentes a los antimicrobianos que el resto de las especies de este grupo.

La resistencia a oxacilina es aún más difícil de detectar en SCN que en $S$. aureus debido a que la colonia es más pequeña y a veces difícil de visualizar en la placa. Además la heterogeneidad de la colonia es aún mayor que en el caso de $S$. aureus ya que el número de microorganismos que expresan resistencia dentro de la colonia es muy bajo. La placa de screening no detecta en forma eficiente a cepas de SCN que son resistentes a oxacilina y por esta razón, este método no se debe usar para evaluar resistencia a oxacilina en SCN. En la actualidad para hacer el estudio de susceptibilidad a oxacilina en SCN, el NCCLS recomienda el uso del método de dilución en caldo o el método de difusión por disco usando los nuevos puntos de corte (Tabla 2).

\section{Estudio de susceptibilidad de Staphylococcus a vancomicina}

Hoy en día las infecciones producidas por cepas resistentes a oxacilina deben ser tratadas con vancomicina. Existe bastante preocupación por parte de los médicos clínicos y de los miembros del control de infecciones intrahospitalarias en tratar de reducir el consumo de vancomicina dentro del hospital.

Como todos sabemos, cepas de S. aureus que presentan resistencia intermedia (CIM mayor o igual a $8 \mu \mathrm{g} / \mathrm{ml}$ ) a vancomicina han sido aisladas de pacientes que en su mayoría han recibido un tratamiento prolongado con vancomicina. Estas cepas también presentan habitualmente una susceptibilidad disminuida a teicoplanina y es por ello que se han denominado GISA que significa " $S$. aureus con resistencia intermedia a glicopéptidos. Las primeras cepas de GISA aisladas de muestras clínicas eran todas SAMR, pero últimamente se han aislado cepas con resistencia intermedia

Tabla 3. Métodos recomendados para la detección de resistencia intermedia a vancomicina (CIM > $8 \mu \mathrm{g} / \mathrm{ml}$ ) en Staphylococcus spp

- La placa de screen (BHI con $6 \mu \mathrm{g} / \mathrm{ml}$ de vancomicina) incubada por $24 \mathrm{hrs}$ puede ser usada como método de screening. Este método necesita confirmación por un método de CIM en cepas que aparecen como resistentes. Además debe ser realizado estricto control de calidad con cepas de E. faecalis ATCC 29212 y con S. aureus ATCC 29213.

- El método recomendado por el NCCLS para confirmar resistencia es el método de microdilución por caldo usando caldo de Mueller-Hinton con ajuste de cationes y una incubación de 24 hrs.

- Métodos automatizados (Microscan, Vitek) detectan resistencia intermedia sólo si se usa incubación de al menos $16 \mathrm{hrs}$. Se recomienda que cepas de $S$. aureus con una CIM de > $4 \mu \mathrm{g} /$ $\mathrm{ml}$ con estos métodos sean consideradas como posiblemente resistentes y deben ser enviadas a un laboratorio de referencia.

- Un E-test® realizado en placas de Mueller-Hinton y una incubación de 24 hrs es también confiable si se siguen las indicaciones del fabricante al leer e interpretar la CIM.

- El método de difusión por disco NO debe ser usado para el estudio de susceptibilidad de Stafilococcus a vancomicina ya que este método no diferencia cepas susceptibles de cepas con resistencia intermedia a vancomicina. 
a vancomicina pero que son susceptibles a oxacilina (SAMS). Este hallazgo complica aún más nuestra tarea en el laboratorio debiéndose revisar cuidadosamente el resultado de vancomicina, incluso en aquellas cepas de SAMS.

El mecanismo exacto de resistencia a vancomicina en $S$. aureus aún no ha sido totalmente aclarado. Las cepas de $S$. aureus con resistencia intermedia aisladas en Japón y en E.U.A., tienen una pared celular gruesa y algunos precursores que participan en la síntesis de la pared están aumentados en cantidad, pero aún no se ha confirmado que estas modificaciones jueguen un papel en la resistencia a vancomicina. Sí se sabe que la resistencia a vancomicina en S. aureus no está mediada por los genes que causan la resistencia a vancomicina en enterococos.

\section{Métodos recomendados para detectar re- sistencia a vancomicina en $S$. aureus}

En la actualidad el NCCLS recomienda el método de la placa de screening para detectar las cepas de $S$. aureus con resistencia intermedia a vancomicina (Tabla 3). El método de difusión por disco no es confiable en la detección de $S$. aureus con resistencia intermedia a vancomicina ya que no diferencia estas cepas de cepas susceptibles. Por este motivo, el NCCLS no recomienda usar este método. En cambio preconiza la placa de screening preparada con agar de infusion cerebro corazón (BHI). Esta placa debe ser incubada durante $24 \mathrm{hrs}$ antes de ser leída. Si una cepa de estafilococo crece en la placa de screening, esta cepa debe ser confirmada como resistente usando el método de dilución en caldo para obtener la CIM (Tabla 3). En general, los métodos automatizados son capaces de detectar estas cepas si la incubación ha sido de al menos 16 hrs, aunque el ideal es usar una incubación de 24 hrs. Sistemas que usan períodos de incubación menor a 16 hrs no son confiables. El E-test ${ }^{\circledR}$ también detecta cepas con resistencia intermedia a vancomicina pero la CIM es generalmente una dilución más baja que con los otros métodos. ${ }^{5}$ Es importante seguir las indicaciones del fabricante para leer e interpretar el crecimiento fino de colonias alrededor de la elipse.
En la actualidad el CDC en E.U.A. recomienda que aquella cepa de $S$. aureus con una CIM a vancomicina $>4 \mu \mathrm{g} / \mathrm{ml}$ sea clasificada como posiblemente resistente a vancomicina debiendo ser enviada a un laboratorio de referencia.

\section{Recomendaciones actuales en el estudio de susceptibilidad en Staphylococcus saprophyticus}

El NCCLS en su edición de enero del 2001 recomendó por primera vez no realizar estudio de susceptibilidad en este microorganismo. Las razón principal para esta recomendación proviene del hecho que muchas cepas de $S$. saprophyticus aparecen como oxacilina resistente in vitro, pero son clínicamente susceptibles. Esto es debido a que este microorganismo es un patógeno aislado sólo en infecciones urinarias y las concentraciones que los $\beta$-lactámicos alcanzan en orina son mucho más altas que las obtenidas en el plasma y por lo tanto estos agentes son capaces de inhibir a las cepas de $S$. saprophyticus con CIMs por sobre el punto de corte. Por este motivo el NCCLS decidió que como las infecciones causadas por este microorganismo responden al tratamiento habitual, no es necesario hacer estudio de susceptibilidad. Sin embargo, ésta no es una recomendacion fácil de poner en práctica en la rutina del laboratorio ya que los médicos clínicos esperan ese informe. Personalmente recomiendo que si se desea hacer el estudio, se prueben aquellos antimicrobianos más comúnmente usados para tratar las infecciones urinarias. Pero debe excluirse oxacilina ya que si el microorganismo es resistente in vitro se debiera infomar resistente a todos los $\beta$-lactámicos (como discutiéramos más arriba con los otros estafilococos), cuando en la práctica la infección urinaria causada por S. saprophyticus todavía puede ser tratada con estos agentes.

En resumen, en este artículo hemos revisado los métodos recomendados para detectar en forma eficiente aquellas cepas de estafilococos resistentes a oxacilina y también hemos revisado los métodos que se pueden usar para detectar y confirmar la presencia de resistencia intermedia a vancomicina. Para una explicación 
más extensa de los otros agentes antimicrobianos que pueden ser estudiados en estafilococos recomiendo revisar las últimas ediciones del NCCLS.

\section{BIBLIOGRAFÍA}

1.- NATIONAL COMMITTEE FOR CLINICAL LABORATORY STANDARDS. Performance standards for antimicrobial susceptibility testing. Twelve informational supplement M100-S12. Wayne, Pennsylvania: NCCLS, 2002.

2.- KIM S D, MCDONALD L C, JARVIS W R et al. Determining the significance of coagulase-negative staphylococci isolated from blood cultures at a commu- nity hospital: a role for species identification. Infect Control Hosp Epidemiol 2000; 21: 213-7.

3.- FASOLA E L, PETERSON L R. Laboratory Detection and Evaluation of Antibiotic-Resistant Staphylococcus aureus Nosocomial Infections. In Advances in Pathology Volume V. 1992. Weinstein [Ed.]. MosbyYear Book, Inc., Chicago, IL, p. 285-306.

4.- TENOVER F C, JONES R N, SWENSON J M, ZIMMER B, MCALLISTER S, JORGENSON J H. for the NCCLS Staphylococcus Working Group. Methods for improved detection of oxacillin resistance in coagulase-negative staphylococci: Results of a multicenter study. J Clin Microbiol 1999; 37: 4051-8.

5.- TENOVER F C, LANCASTER M V, HILL B, STEWARD C D et al. Characterization of staphylococci with reduced susceptibilities to vancomycin and other glycopeptides. J Clin Microbiol 1998; 36: 1020-7.

Correspondencia a:

Elizabeth Palavecino Rosales

Email: epalavecino@hotmail.com 